\title{
Exstrophy-epispadias complex
}

INSERM

\section{Source}

INSERM. (1999). Orphanet: an online rare disease and orphan drug data base. Exstrophyepispadias complex. ORPHA:322

Exstrophy-Epispadias Complex (EEC) represents a spectrum of genitourinary malformations ranging in severity from epispadias (E) and classical bladder exstrophy (CEB) to exstrophy of the cloaca (EC) as the most severe form (see these terms).

Depending on severity, the EEC may involve the urinary system, the musculoskeletal system, the pelvis, the pelvic floor, the abdominal wall, the genitalia and sometimes the spine and the anus. 